University of Nebraska - Lincoln

DigitalCommons@University of Nebraska - Lincoln

Valery Forbes Publications

Papers in the Biological Sciences

2011

\title{
Adding Value to Ecological Risk Assessment with Population Modeling
}

Valery E. Forbes

University of Nebraska-Lincoln, veforbes@umn.edu

Peter Calow

Roskilde University, pcalow2@unl.edu

Volker Grimm

Helmholtz Center for Environmental Research, Leipzig, Germany

Takehiko I, Hayashi

National Institute for Environmental Studies, Tsukuba, Ibaraki, Japan

Tjalling Jager

Vrije Universiteit Amsterdam

See next page for additional authors

Follow this and additional works at: https://digitalcommons.unl.edu/biosciforbes

Part of the Pharmacology, Toxicology and Environmental Health Commons

Forbes, Valery E.; Calow, Peter; Grimm, Volker; Hayashi, Takehiko I,; Jager, Tjalling; Katholm, Agnete; Palmqvist, Annemette; Pastorok, Rob; Salvito, Dan; Sibly, Richard; Spromberg, Julann; Stark, John; and Stillman, Richard A., "Adding Value to Ecological Risk Assessment with Population Modeling" (2011). Valery Forbes Publications. 4.

https://digitalcommons.unl.edu/biosciforbes/4

This Article is brought to you for free and open access by the Papers in the Biological Sciences at DigitalCommons@University of Nebraska - Lincoln. It has been accepted for inclusion in Valery Forbes Publications by an authorized administrator of DigitalCommons@University of Nebraska - Lincoln. 


\section{Authors}

Valery E. Forbes; Peter Calow; Volker Grimm; Takehiko I, Hayashi; Tjalling Jager; Agnete Katholm;

Annemette Palmqvist; Rob Pastorok; Dan Salvito; Richard Sibly; Julann Spromberg; John Stark; and

Richard A. Stillman 


\title{
Perspective
}

\section{Adding Value to Ecological Risk Assessment with Population Modeling}

\author{
Valery E. Forbes, ${ }^{1}$ Peter Calow, ${ }^{1}$ Volker Grimm, ${ }^{2}$ Takehiko I. Hayashi, ${ }^{3}$ \\ Tjalling Jager, ${ }^{4}$ Agnete Katholm, ${ }^{1}$ Annemette Palmqvist, ${ }^{1}$ Rob Pastorok, ${ }^{5}$ \\ Dan Salvito, ${ }^{6}$ Richard Sibly, ${ }^{7}$ Julann Spromberg, ${ }^{8}$ John Stark, ${ }^{9}$ \\ and Richard A. Stillman ${ }^{10}$ \\ ${ }^{1}$ Department of Environmental, Social and Spatial Change, Roskilde University, \\ Roskilde, Denmark; ${ }^{2}$ Helmholtz Center for Environmental Research-UFZ, \\ Department of Ecological Modelling, Leipzig, Germany; ${ }^{3}$ Research Center for \\ Environmental Risk, National Institute for Environmental Studies, Tsukuba, \\ Ibaraki, Japan; ${ }^{4}$ Department of Theoretical Biology, Vrije Universiteit Amsterdam, \\ Amsterdam, The Netherlands; ${ }^{5}$ Integral Consulting Inc., Woodinville, WA, USA; \\ ${ }^{6}$ Research Institute for Fragrance Materials, Woodcliff Lake, NJ, USA; ${ }^{7}$ Department \\ of Biological Sciences, University of Reading, Reading, UK; ${ }^{8} \mathrm{NOAA}$ Fisheries \\ Service, Northwest Fisheries Science Center, Seattle, WA, USA; ${ }^{9}$ Washington State \\ University, Puyallup Research and Extension Center, Puyallup, WA, USA; ${ }^{10}$ School \\ of Conservation Sciences, Bournemouth University, Talbot Campus, \\ Poole, Dorset, UK
}

\begin{abstract}
Current measures used to estimate the risks of toxic chemicals are not relevant to the goals of the environmental protection process, and thus ecological risk assessment (ERA) is not used as extensively as it should be as a basis for cost-effective management of environmental resources. Appropriate population models can provide a powerful basis for expressing ecological risks that better inform the environmental management process and thus that are more likely to be used by managers. Here we provide at least five reasons why population modeling should play an important role in bridging the gap between what we measure and what we want to protect. We
\end{abstract}

Received 7 March 2010; accepted 10 March 2010.

The views expressed here represent those of the authors and not necessarily those of the individual participants at the RUC09 Workshop.

Address correspondence to Valery Forbes, School of Biological Sciences, University of Nebraska Lincoln, 348 Manter Hall, Lincoln, NE, 68588-0118, USA. E-mail: vforbes@unl.edu 


\section{E. Forbes et al.}

then describe six actions needed for its implementation into management-relevant ERA.

Key Words: ecological risk assessment, population modeling, environmental management, extinction risk, extrapolation, socioeconomic analysis.

\section{ERA CAN FAIL TO INFORM RISK MANAGEMENT}

Ecological risk assessment (ERA) is not used as extensively as it should be as a basis for cost-effective management of environmental resources (Landis 2009). One fundamental reason for this is that our measures of risk are not relevant to the goals of the environmental protection process. For example, the data used to estimate the likelihood of adverse ecological effects of toxic chemicals in the environment typically include responses of survival, growth, or reproduction of individuals measured after a specific exposure duration under constant and typically favorable laboratory conditions. But these organism-level endpoints are far removed from the ecological features that the process aims to protect (i.e., the long-term persistence of populations of species in space and time under naturally varying field conditions and in the presence of other stressors). For toxic chemical assessments, the focus of this article, ecological risk is most often characterized as a ratio of predicted or measured exposure to predicted no-adverse-effect level expressed as a concentration or dose (in the EU this is specified as a risk characterization ratio-RCR). RCRs can provide useful screening tools when exposure and no-effect levels are calculated using appropriately conservative assumptions. However, they suffer from a number of disadvantages, not the least of which is that their relationship to the likelihood and degree of ecological impacts (i.e., risk) is unknown. Similarly, risk management often involves socioeconomic analyses, and yet the hazard ratios that have dominated risk assessment are not readily used or usable in the ecological valuation process. For large classes of chemicals (e.g., pharmaceuticals, pesticides) and those with specific chemical or biological properties (e.g., PBTs, endocrine disruptors) the present paradigm is insufficient and may be either under or over protective. But without a better assessment considering population-level effects in light of values ascribed from socioeconomic analysis, we cannot provide a sufficient level of understanding/assurance that could make ERA relevant for management decisions.

Appropriate population models can provide a powerful basis for expressing ecological risks that better inform the environmental management process and thus are more likely to be used by managers. Whereas population modeling has been used extensively in conservation biology and other types of ecological management, its use in chemical risk assessment has been minimal. Several recent workshops have focused on the potential for implementing population modeling in chemical risk assessment. The LEMTOX Workshop, held in Leipzig (Germany) in 2007 focused specifically on the use of modeling for pesticide registration and risk assessment in the EU (Forbes et al. 2009; Thorbek et al. 2010); one important outcome of this workshop was the EU-funded Marie Curie Training Network "CREAM," where both specific models and general guidance for good modeling practice will be developed (Grimm et al. 2009; http://cream-itn.eu). The Risk Assessment Forum Technical Workshop on Population-Level Ecological Risk Assessment, held in Washington 
(DC, USA) in 2008 was concerned with developing guidance for U.S. Environmental Protection Agency (USEPA) programs (USEPA 2009).

The present Perspective describes the outcome of the Roskilde Workshop on Integrating Population Modeling into Ecological Risk Assessment (RUC09) that specifically reviewed the current state of the science and identified research needed to facilitate the implementation of population modeling in ERA. The key features of RUC09 that made it different from the workshops listed above were: (a) it covered industrial chemicals in general; (b) it was not specific for a particular jurisdiction; and (c) it developed a set of concrete actions for the implementation of population modeling in regulatory decision-making. In this Perspective we first explicitly address how population modeling adds value to ERA and then describe the actions needed for its implementation into management-relevant ERA.

\section{POPULATION MODELING ADDS NEEDED VALUE TO ERA}

ERA involves extrapolating from limited observations to ecological responses that are broadly related to likely impairments in biodiversity and ecosystem processes and services. A major theme in what follows is that population modeling should, for at least the following five reasons, play an important role in bridging the gap between what we measure and what we want to protect (Forbes et al. 2008):

Reason 1: Population modeling reduces uncertainty in extrapolation of standard test results to ecologically relevant impacts. Standard toxicity tests typically measure impacts of chemicals on individual survival, reproduction or growth. In many cases, only one of these measures of effect is used to assess risk, and even when multiple endpoints are used, attempts to integrate the results across endpoints are qualitative or semi-quantitative (scoring) at best. Using results for multiple endpoints in even a simple population model can lead to unexpected outcomes regarding the consequences of impacts of toxicants on individual-level traits for population dynamics. For example, Pedersen et al. (2009) found that exposure to the polycyclic musk, HHCB, resulted in a $43 \%$ decline in reproductive output of the freshwater snail, Potamopyrgus antipodarum, but that this decline in fecundity led to a less than $2 \%$ impact on population growth rate. On the other hand, using matrix population models incorporating density dependence, Hayashi et al. (2009) showed that populationlevel effects as indicated by the percentage reduction in equilibrium population size often exceeded the percentage reductions in vital rates of individuals.

Reason 2: Population modeling can identify high-risk scenarios for which testing efforts can be prioritized. It is well established that the extent to which individuallevel responses impact population dynamics depends strongly on both the status of the population (i.e., in growth phase, equilibrium or decline) and the degree of density-dependence in survival, growth, and reproduction (Calow et al. 1997; Grant 1998; Hayashi et al. 2009). Hence, the same magnitude of effects on individual-level endpoints under different conditions or in different species under similar conditions is likely to lead to markedly different population outcomes. Also, extrapolating from particular ecological settings (as in mesocosms) to other ecological settings may be crucially influenced by features such as habitat complexity and various biotic and abiotic conditions. In principle, such features can be manipulated in population models and their relative importance for population risk determined (Grimm 
and Railsback 2005; van den Brink et al. 2007). For example, Wang and Grimm (2010) used an individual-based population model of the common shrew to explore population-level effects of a hypothetical pesticide under different application scenarios. Model organisms were exposed on one certain day every year over a period of 30 years and as a result experienced 10 or $20 \%$ adult mortality on that day. If exposure occurred on April 1, when the animals started to reproduce, populations continuously declined to extinction in a landscape without hedges but stabilized at a moderately lowered equilibrium in landscapes including hedges. Hedge habitats provided greater food densities, which somewhat mitigated the effects of both density dependence and pesticides. If exposure occurred on July 15, when the population reached highest densities, populations also declined to extinction in landscapes including hedges.

Reason 3: Population modeling provides outputs that are essential for choosing among management alternatives and for facilitating cost-benefit assessments. Population models can link the generalized protection goals stated in environmental legislation with more operational goals that aim to protect ecosystem services (Maltby et al. 2009). These services invariably depend on particular populations, and hence values are most directly ascribed to changes in population attributes (e.g., abundance, age/stage structure, population growth rate, realized equilibrium population size). Under the EU Existing Substances Regulation (precursor to REACH), many of the risk reduction strategies for ecological protection could not be based upon cost-benefit analysis because the ERAs did not lead to a specification of ecological units that could be valued; that is, they were in terms of RCRs (IMV 2007). In contrast to management of toxic chemicals, management decisions about natural resource conservation and harvest rely on population-level endpoints and (at least in the recent past) have made appropriate use of population models. For example, conservation biologists have applied models for population viability analyses to evaluate the status of populations and risk of their extinction. Output from these analyses is directly related to the protection goals of legislation like the Endangered Species Act in the United States.

Population modeling can integrate the effects of multiple stressors, which is impossible in any meaningful way with RCRs. For example, An et al. (2009) evaluated the effects of male feminization due to exposure to endocrine disrupting chemicals (EDCs) on roach (Rutilus rutilus) populations in European rivers. Changes in population growth rate and extinction risk due to feminization were evaluated in populations with and without fishing mortality. In the presence of selective fishing, feminization of males significantly increased the extinction risk of modeled roach populations and would cause population extinction in some rivers (e.g., the Volga Delta and Ural Rivers) with high rates $(>90 \%)$ of intersex. But without fishing pressure, even $100 \%$ intersex would be unlikely to lead to population extinction in modeled roach populations (An et al. 2009). These authors provided information that could be used directly by managers of local fisheries.

Reason 4: Population modeling reduces the use of animal testing in a more ecologically sound manner than relying on in vitro or quantitative structure activity relationship (QSAR) methods. There is enormous societal pressure to reduce animal testing and yet reductionist in vitro and QSAR techniques may lead to outcomes that either 
underestimate or overestimate the effects of substances at relevant ecological levels (Forbes et al. 2008; Hartung 2009). Population modeling can provide more realistic outcomes by incorporating important mechanistic understanding. For example, Dalkvist $e t a l$. (2009) simulated the impacts of a pesticide having hormone-disrupting and epigenetic effects on vole populations. Using a sophisticated agent-based model coupled to a geographic information system (GIS) database, they showed that the long-term risk to vole populations was relatively insensitive to the toxicological sensitivity of the voles to the pesticide (i.e., the lowest-observed-effect-level (LOEL)) and much more sensitive to assumptions about exposure. In addition, the model allowed realistic simulations of population-level impacts over a much longer time scale (i.e., $>100$ years) than could have been tested empirically.

It is often of interest to assess risks to protected or endangered species. For ethical reasons there may be restrictions on the quantity and type of laboratory testing permitted on such species, and in such cases application of population modeling can make the most out of limited test data. For example, Baldwin et al. (2009) investigated the population-level effects of acetylcholinesterase (AChE) inhibiting pesticides on endangered Chinook salmon. Limited laboratory studies demonstrated that sublethal reductions of AChE activity resulted in decreased swimming and feeding behaviors. These observed relationships were built into an individual-based growth model linking exposure to size. Field studies provided a size-dependent survival relationship during the freshwater residence of juvenile salmon, where pesticide exposure could occur. The resulting survival was fed into a life-history matrix model to determine changes in population abundance due to the sublethal pesticide exposures.

Reason 5: Population modeling can introduce ecological complexity into risk assessment in a cost-effective manner. ERAs are often carried out in a tiered way and move from simple laboratory tests to complex mesocosms/field tests incorporating increased ecological realism and thus complexity. These kinds of high-tier tests are not practical for all situations and are expensive and time consuming. Whereas it can be costly to develop complex population models, once produced they can be available for repeated use at minimal cost. For example, a number of studies investigating the effects of spatial complexity in exposure scenarios indicate that inter-individual variability in exposure can either increase (Palmqvist and Forbes 2008) or reduce (Dalkvist et al. 2009) risk compared to the standard homogeneous exposure scenarios typically assumed in risk assessment. Likewise, by incorporating realistic information on the species' ecology into a population model, Baldwin et al. (2009) were able to show that the change in size when fish migrated to the ocean caused a reduction in individual survival over successive years and thereby reduced the population growth rate.

Current approaches to ERA assume that the susceptibility of species to toxic chemicals is defined entirely by toxicological responses whereas the importance of life history is ignored. However, the same impact on individual-level traits in different species may result in very different population-level outcomes due to differences in life-history strategies (Calow et al. 1997; Stark et al. 2004). Thus simply comparing mortality among species does not provide enough information to determine the relative susceptibility of populations of different species when exposed to toxic chemicals (or other stressors). 


\section{E. Forbes et al.}

In addition to their value in adding ecological complexity into risk assessments, population models are amenable to probabilistic analyses. Outputs can be easily converted to quantitative risk estimates. Uncertainties can be evaluated in a more meaningful way than is currently done under the RCR approach.

\section{THE PATH FORWARD-ACTIONS NEEDED}

Although there are a number of good reasons (see above) for integrating population modeling into ERA, so far its application has been limited due to a general lack of guidance regarding what models of which species to use, coupled with a lack of appropriate case studies demonstrating the added value of population modeling for ERA (Thorbek et al. 2010). Another barrier to the use of population models is uncertainty as to which population endpoints are most appropriate for risk assessment and in particular as inputs into socioeconomic assessments. Actions 1 and 2, below, provide concrete examples of how guidance for population modeling in risk assessment could be developed. Action 3 analyzes the relative strengths and weaknesses of different population-level endpoints for risk assessment. Actions 4, 5, and 6 provide a basis for case studies showing how population modeling can add value in terms of extrapolating from individuals to populations, across species and from laboratory to field, respectively.

Action 1: Develop a decision framework for applying population models in risk assessment and risk management for chemical regulatory programs. There is a wide range of models already available for deployment for ERA (Barnthouse et al. 2008; Forbes et al. 2008; Pastorok et al. 2001; USEPA 2009; Thorbek et al. 2010). However, guidance is needed for applying population models in different regulatory contexts. New tools for value-relevant decision-making and risk communication of population risk expressions are required. A decision framework for selecting specific types of models for defined risk management issues should be the focal point of such guidance. In the decision framework, risk management issues should be linked to regulatory and risk assessment objectives, ecological endpoints, population model outputs, and related risk expressions. Model selection criteria need to be defined to optimize the value added by population modeling. Support tools such as model standardization, validation, and design protocols will also be required. As a common language for formulating these designs, the recently developed ODD protocol (Overview, Design concepts, Details) for communicating individual-based models (Grimm et al. 2006) would greatly facilitate model development. A generalized format for documenting not only models but also their development and analysis will be developed in the CREAM project (http://cream-itn.eu; Schmolke et al., unpubl. manuscript). Risk communication tools for use by managers in presenting the results of population models used in an ERA should also be developed.

Action 2: Develop generic models of many species and environments. Many detailed population models have been developed for particular species in particular ecological circumstances. Risk assessment would be facilitated however by the development of a suite of generic models that can be tailored for the range of circumstances that apply in various risk contexts. Such generic models have already been developed in other management contexts, for example for stream fish (Railsback 
and Harvey 2002), coastal birds (Stillman 2008), and tropical forests (Huth et al. 2005). The common feature of these models is that they are based on what individuals do, and this is known either directly from evidence or from "first principles." Examples include the ways in which individuals make adaptive decisions regarding key behaviors like foraging or habitat selection, individual physiology (Peterson et al. 2008), and photosynthesis (Huth et al. 2005).

Stillman (2008) presented a general design for the individual-based modeling of mortality in foraging animal populations that has been widely applied to coastal birds (Stillman and Goss-Custard 2010). Similar designs, using the ODD approach described under Action 1 could be developed for other species and guilds. The effects of the chemical on individuals can be modeled using simple transparent extrapolation from existing laboratory data, based on the individual's metabolic weight and life history. Model design should focus on first principles and on using parameters that can directly be observed and measured. Then, the same approach of identifying appropriate first principles, general model designs, and selecting representative species and environments, might be applied to all the major taxonomic groups included in ERA.

Action 3: Select value-relevant population endpoints for risk assessment. By valuerelevant endpoints we mean endpoints that relate to the long-term persistence of populations. These are important because of their contribution to ecosystem services and because they can therefore be given monetary value through socioeconomic analysis. It will be necessary to define the most appropriate population-level endpoints in the context of the Decision Framework described earlier. A variety of endpoints (e.g., population growth rate, equilibrium population density, age/size structure, extinction risk) can be derived from the major classes of population models applied to different types of risk assessment scenarios. It will also be necessary to validate the models by performing life table response experiments to identify which population-level endpoints are best able to predict the long-term persistence of populations in space and time. These kinds of experiments will inevitably involve species with short generation times so that long-term responses over multiple generations can be estimated. For each species and model there should be a systematic consideration of how the various population-level endpoints vary in terms of sensitivity to chemicals (i.e., percent change relative to reference conditions), statistical detectability of effect, ability to predict the dynamics of intact populations, and their ease of use in socioeconomic analyses. For example, Wang and Grimm (2010) compared different population-level endpoints (population growth rate, population size, extinction risk) and explored the statistical detectability of population-level effects. One of their findings was that effects can be easier to detect in populations that are in fact less at risk, simply because they live in a more benign habitat that allows for higher and less variable population sizes.

Action 4: Use population models to facilitate the extrapolation from individuallevel endpoints to population-level effects. Standard toxicity tests usually focus on effects on individual-level endpoints such as survival, growth and reproduction, after a fixed exposure period. Simple summary statistics such as effect concentrations for some fraction of the population differ between endpoints, depend on how the endpoint is expressed (e.g., body weight or length), and change in time in a manner that depends on the chemical, the species, and the endpoint of interest (e.g., Álvarez 


\section{E. Forbes et al.}

et al. 2006). It is therefore not surprising that the effects on a single endpoint, after a single test duration, are not predictive of population-level effects (Forbes and Calow 1999). In many risk assessments the no observed effect concentration for individuallevel responses is used to characterize risk, and this is likely to be over-protective for population-level effects. In order to improve the extrapolation from toxicity patterns for all individual-level endpoints over the life cycle of the organism to impacts on populations, the individual endpoints should be integrated into a population model. In particular, prediction of the ecological consequences of substances such as EDCs that have non-monotonic dose-response relationships, and substances that show very different dose-response relationships for different individual-level traits, could be substantially improved using population modeling techniques.

The use of population models does not necessarily imply an increased testing effort. To demonstrate the feasibility and benefits of various modeling approaches for risk assessment, a systematic analysis of different types of population models-from very simple to very complex-could be applied to standard toxicity test data. In this way both the data requirements of different model types, and their performance in terms of providing outputs relevant for ERA, can be assessed. As a start, data gathered according to current test protocols could be used as input to very simple population models (e.g., Euler-Lotka, simple matrix models (Sibly and Calow 1986) ) to quantify the links between individual-level responses and population-level responses (e.g., Stark et al. 2004; Palmqvist and Forbes 2008). In fact, the input data required for (simple) population models are already being provided by toxicity tests performed according to several standard test protocols. For example, the OECD (1998) 21-day Daphnia protocol prescribes that survival and reproduction should be determined at least three times a week, input that can directly fuel a population projection. Additional biological and ecological relevance could be incorporated by using more complex models (e.g., density-dependent matrix models, metapopulation models, and individual-based models). Based on the data needs of the models, guidance may be necessary on optimizing the test protocols to provide the needed parameters.

Action 5: Use population models to extrapolate from test species to untested species. Each species evolves a life-history strategy adapted to sustaining viable populations in their natural habitat. Resource availability and survival potential influence the time and energy invested in each life stage (i.e., embryo, larva, juvenile, and adult stages). As an expression of adaptation, energy allocation tradeoffs between growth and reproduction tend to maximize the individual's reproductive contribution to future generations. Adaptations to local environmental factors, such as seasonal conditions and severity of stochastic impacts, occur over many generations and lead to the evolution of numerous variations in life-history strategies (Winemiller and Rose 1992). Each strategy provides for continuity of generations under normal environmental conditions; however, some strategies may confer greater or lesser susceptibility to anthropogenic perturbations that fall outside of the species' adaptive history. A fish species that reproduces multiple times each year may more easily compensate for a toxic impact than an annual spawner since it may have unaffected fry from previous spawning events, or eggs to be spawned later in the season. This illustrates how population responses to chemical stressors may differ depending on 
the various life-history characteristics exhibited (Stark et al. 2004; Spromberg and Birge 2005).

Population models can be used to assess how differences in physiological susceptibility across life stages can translate into population-level impacts (i.e., change in population growth rate, abundance, age/stage distribution, probability of extinction, time to recovery). The outputs of such models can investigate whether commensurate impacts to juvenile stages, adult stages, or reproduction will result in the same population-level impacts within and across life-history strategies. These studies would also contribute to Action 2, above, to develop a set of general models across invertebrates, plants, fish, birds, and mammals that include common toxicity test species, commercially or culturally important species, and rare species. The outputs of such an effort could include a list of life-history or life-cycle characteristics that confer susceptibility and a list of species within each class of organisms that could be used as surrogates depending on the requirements of the risk assessment.

Action 6: Use population models to add ecological realism for laboratory-to-field extrapolation. The distribution of chemicals in the environment is not homogenous. Chemical concentrations in the field often, if not always, vary both in time and space. However, current laboratory test procedures are designed to keep chemical exposures constant over time and to expose all individuals to the same homogenous chemical concentration. In fact, in standard procedures we go to great lengths to assure that the chemicals are homogenously distributed in the exposure medium, and to renew exposure media if the chemical concentration is expected to decrease over time. This is in sharp contrast to the field where chemical inputs may vary seasonally (e.g., pesticides), as pulses following runoff events or accidents, and spatially (e.g., with distance from point sources, due to preferential association with certain environmental constituents such as organic matter in sediments). In addition to keeping the exposure concentrations constant, both in time and space, standard laboratory toxicity tests also aim to provide optimal conditions (except for the tested contaminant) for the exposed organisms. In a more realistic field scenario, conditions are not optimal and, for example, organisms in the field will often be under the control of density constraints, suppressing population growth even in the absence of contaminants. These density effects may or may not be compensatory to the effects of chemicals.

Metapopulation simulations have shown that risk from chemicals or other stressors may be underestimated when ignoring spatial heterogeneity in a chronic exposure scenario, but that the degree to which this is the case is highly dependent on the dispersal ability and habitat selection behavior of the species in question (Palmqvist and Forbes 2008; Walters et al. 2008). The resulting patterns of risk are likely to be scale dependent (i.e., to depend on how organisms with different sizes or mobility experience differences in habitat complexity, and how such affect the risk assessment of chemicals). It is obvious that the same landscape, and the patchiness within it, will be perceived very differently by a skylark and a beetle (Nabe-Nielsen et al. 2010), but also within a single population there may be differences in how different life stages sense the environment. For example, a slowly moving adult polychaete or a sessile adult bivalve in the subtidal zone will probably experience less habitat heterogeneity compared to their free-swimming larvae. In contrast, the free-swimming larvae are 


\section{E. Forbes et al.}

better able to avoid suboptimal habitat compared to the slowly moving or sessile adults. Therefore, laboratory tests that assume constant and identical exposure to all individuals in a population lack ecological realism and potentially misrepresent the risks associated with exposure to toxic chemicals.

A combination of metapopulation and individual-based models could be used to explore the importance of spatial and temporal heterogeneity in chemical exposure to population-level risk. Scenarios could be developed that are based on the most common types of spatial and temporal heterogeneity in chemical exposure, selected representative life-cycle types and different population densities to identify the conditions under which ignoring exposure heterogeneity leads to overestimates and underestimates of risk.

\section{CONCLUSIONS}

Current approaches for risk assessment typically have low ecological relevance (e.g., the RCR approach), which can compromise regulatory decisions based on such assessments and lead to either substantial underestimation or substantial overestimation of risk. The application of population models adds value to ERA by providing outputs that are more directly related to management protection goals. We note in particular that the susceptibility of populations to chemical stressors is more complicated than predicted simply on the basis of the toxic properties of a compound or the physiology of the exposed organisms. Also, utilizing population models in ERA will provide regulators with value-relevant endpoints for decisionmaking and protection-if done properly. Finally, whereas ecologically realistic experiments are both expensive and time consuming, structurally realistic population models (Wiegand et al. 2003; Grimm et al. 2005) that have been thoroughly designed, tested, and analyzed (and that include uncertainty analysis) can be rerun numerous times to test different hypotheses at very low cost (e.g., Dalkvist et al. 2009; Wang and Grimm 2010)

There is no shortage of available and accessible population models. Recently, there has been a considerable amount of activity to promote population modeling in ERA (Akçakaya et al. 2008; Barnthouse et al. 2008), and as evidenced by the outcomes of workshops discussed herein. Yet there has been inertia in the acceptance and use of this approach by regulators. By developing guidance on using population models that is relevant to regulatory programs (e.g., generic models and model designs, with model selection criteria defined within a Decision Framework), it is possible to provide tools ready for use in risk assessment for a wide range of species and environments within a reasonable timeframe. Successful implementation will require transparent, robust, and well-tested population models that are applicable for assessing the risks of chemicals in different legislative contexts. The actions outlined here are intended to facilitate the effective implementation of population modeling in ERA by the development of specific guidance and concrete case studies.

\section{ACKNOWLEDGMENTS}

Funding was provided by the Research Institute for Fragrance Materials (RIFM), ECETOC, and Roskilde University. The authors also thank the participants of the 
RUC09 Workshop for the stimulating discussions that led to the production of this article.

\section{REFERENCES}

Akçakaya HR, Stark JD, and Bridges TS. 2008. Demographic Toxicity: Methods in Ecological Risk Assessment. Oxford University Press, Oxford, UK

Alda Álvarez O, Jager T, Nuñez Coloa B, et al. 2006. Temporal dynamics of effect concentrations. Environ Sci Technol 40:2478-84

An W, Hu JY, Giesy JP, et al. 2009. Extinction risk of exploited wild roach (Rutilus rutilus) populations due to chemical feminization. Environ Sci Technol 43:7895-901

Baldwin DB, Spromberg JA, Collier TK, et al. 2009. A fish of many scales: Extrapolating sublethal pesticide exposures to the productivity of wild salmon populations. Ecol Appl 19: 2004-15

Barnthouse LW, Munns WR Jr, and Sorensen MT (eds). 2008. Population-Level Ecological Risk Assessment. CRC Press, Boca Raton, FL, USA

Calow P, Sibly R, and Forbes VE. 1997. Risk assessment on the basis of simplified population dynamics' scenarios. Environ Toxicol Chem 16:1983-9

Dalkvist T, Topping CJ, and Forbes VE. 2009. Population-level effects of pesticide-induced chronic effects on individuals depend more on ecology than toxicology. Ecotox Environ Safe 72:1663-72

Forbes VE and Calow P. 1999. Is the per capita rate of increase a good measure of populationlevel effects in ecotoxicology? Environ Toxicol Chem 18:1544-56

Forbes VE, Calow P, and Sibly RM. 2008. The extrapolation problem and how population modeling can help. Environ Toxicol Chem 27:1987-94

Forbes VE, Hommen U, Thorbek P, et al. 2009. Ecological models in support of regulatory risk assessments of pesticides: developing a strategy for the future. Integr Environ Assess Manag 5:167-72

Grant A. 1998. Population consequences of chronic toxicity: Incorporating density dependence into the analysis of life table response experiments. Ecol Model 105:325-35

Grimm V and Railsback SF. 2005. Individual-Based Modelling and Ecology. Princeton University Press, Princeton, NJ, USA

Grimm V, Revilla E, Berger U, et al. 2005. Pattern-oriented modeling of agent-based complex systems: Lessons from ecology. Science 310:987-91

Grimm V, Berger U, Bastiansen F, et al. 2006. A standard protocol for describing individualbased and agent-based models. Ecol Model 198:115-26

Grimm V, Ashauer R, Forbes V, et al. 2009. CREAM: A European project on mechanistic effect models for ecological risk assessment of chemicals. Environ Sci Pollut Res 16:614-17

Hartung T. 2009. Toxicology for the twenty-first century. Nature 460:208-12

Hayashi TI, Kamo M, and Tanaka Y. 2009. Population-level ecological effect assessment: Estimating the effect of toxic chemicals on density-dependent populations. Ecol Res DOI 10.1007/s11284-008-0561-6

Huth A, Drechsler M, and Köhler P. 2005. Using multicriteria decision analysis and a forest growth model to assess impacts of tree harvesting in Dipterocarp lowland rain forests. Forest Ecol Manag 207:215-32

IMV (Environmental Assessment Institute). 2007. Challenges for Economic Analysis under REACH. What Can We Learn From Previous Experience? ISBN: 87-7992-050-0. Copenhagen, Denmark

Landis WG. 2009. Why has ecological risk assessment found such limited application? Hum Ecol Risk Assess 15:849-57 


\section{E. Forbes et al.}

Maltby L, Paetzold A, and Warren P. 2009. Sustaining industrial activity and ecological quality: The potential role of an ecosystem services approach. In: Batty LC and Hallberg KB (eds), Ecology of Industrial Pollution: Remediation, Restoration and Preservation. Cambridge University Press, Cambridge, UK

Nabe-Nielsen J, Sibly R, Forchhammer MC, et al. 2010. The effects of landscape modifications on the long-term persistence of animal populations. PLoS ONE 5(1):e8932

OECD (Organisation for Economic Cooperation and Development). 1998. OECD guideline for Testing of Chemicals 211. Daphnia magna reproduction test. Paris, France

Palmqvist A and Forbes VE. 2008. Demographic effects of the polycyclic aromatic hydrocarbon, fluoranthene, on two sibling species of the polychaete, Capitella capitata. In: Akcakaya R, Stark JD, and Bridges TS (eds), Demographic Toxicity: Case Studies in Ecological Risk Assessment. Oxford University Press, Oxford, UK

Pastorok RA, Bartell SM, Ferson S, et al. (eds). 2001. Ecological Modeling in Risk Assessment. Lewis Publishers, Boca Raton, FL, USA

Pedersen S, Selck H, Salvito D, et al. 2009. Effects of the polycyclic musk, HHCB, on individual- and population-level endpoints in Potamopyrgus antipodarum. Ecotox Environ Safe 72:1190-9

Peterson JH, DeAngelis DL, and Paukert CP. 2008. An overview of methods for developing bioenergetic and life history models for rare and endangered species. Trans Am Fish Soc 137:244-53

Railsback SF and Harvey BC. 2002. Analysis of habitat-selection rules using an individual-based model. Ecology 83:1817-30

Sibly RM and Calow P. 1986. Physiological Ecology of Animals. Blackwell Scientific Publications, Oxford, UK

SprombergJA and Birge WJ. 2005. Modeling the effects of chronic toxicity on fish populations: The influence of life-history strategies. Environ Toxicol Chem 24:1532-40

Stark JD, Banks JE, and Vargas R. 2004. How risky is risk assessment: The role that life strategies play in susceptibility of species to stress. Proc Natl Acad Sci USA 101:732-6

Stillman RA. 2008. MORPH-An individual-based model to predict the effect of environmental change on foraging animal populations. Ecol Model 216:265-76

Stillman RA and Goss-Custard JD. 2010. Individual-based ecology of coastal birds. Biol Rev 85:413-434

Thorbek P, Forbes V, Heimbach F, et al. (eds). 2010. Ecological Models for Regulatory Risk Assessments of Pesticides: Developing a Strategy for the Future. Society of Environmental Toxicology and Chemistry (SETAC) and CRC Press, Pensacola and Boca Raton, FL, USA

USEPA (US Environmental Protection Agency) 2009. Summary Report. Risk Assessment Forum Technical Workshop on Population-Level Ecological Risk Assessment. EPA/100/R09/006. Washington, DC, USA

Van Den Brink PJ, Baveco JM, Verboom J, et al. 2007. An individual-based approach to model spatial population dynamics of invertebrates in aquatic ecosystems after pesticide contamination. Environ Toxicol Chem 26:2226-36

Walters S, Kuhn A, Nicholson MC, et al. 2008. Stressor impacts on the common loons in New Hampshire, USA. In: Akcakaya R, Stark JD, and Bridges TS (eds), Demographic Toxicity: Case Studies in Ecological Risk Assessment. Oxford University Press, Oxford, UK

Wang M and Grimm V. 2007. Home range dynamics and population regulation: An individualbased model of the common shrew. Ecol Model 205:397-409

Wang M and Grimm V. 2010. Population models in pesticide risk assessment: Lessons for assessing population-level effects, recovery, and alternative exposure scenarios from modelling a small mammal. Environ Toxicol Chem 29:1292-1300 


\section{ERA Needs More Ecological Relevance}

Wiegand J, Jeltsch F, Hanski I, et al. 2003. Using pattern-oriented modeling for revealing hidden information: A key for reconciling ecological theory and application. Oikos 100:209-22

Winemiller KO and Rose KA. 1992. Patterns of life-history diversification in North American fishes: Implications for population regulation. Can J Fish Aquat Sci 49:2196-218 